\title{
Persistent current in superconducting nanorings
}

\author{
K.A. Matveev, ${ }^{1}$ A.I. Larkin, ${ }^{2}$ and L.I. Glazman ${ }^{2}$ \\ ${ }^{1}$ Department of Physics, Duke University, Box 90305, Durham, NC 27708 \\ ${ }^{2}$ Theoretical Physics Institute, University of Minnesota, Minneapolis, MN 55455
}

(Dated: July 10, 2002)

\begin{abstract}
The superconductivity in very thin rings is suppressed by quantum phase slips. As a result the amplitude of the persistent current oscillations with flux becomes exponentially small, and their shape changes from sawtooth to a sinusoidal one. We reduce the problem of low-energy properties of a superconducting nanoring to that of a quantum particle in a sinusoidal potential and show that the dependence of the current on the flux belongs to a one-parameter family of functions obtained by solving the respective Schrödinger equation with twisted boundary conditions.

PACS numbers: 74.50.+r, 73.23.Ra, 73.63.Nm
\end{abstract}

The properties of superconducting grains change dramatically when their size shrinks to a few nanometers. This phenomenon has recently generated a lot of interest [1]. In particular, it has been established both theoretically and experimentally that the superconductivity cannot be observed if the quantum level spacing in a nanoparticle exceeds the superconducting gap $\Delta$. The recent success in manufacturing of superconducting nanowires [2] has raised similar questions regarding the superconductivity in one-dimensional objects. It has been demonstrated experimentally [3] that as the nanowire becomes thiner, the superconducting transition in it disappears, and a finite resistance is observed at low temperatures. The suppression of the superconductivity in thin wires was attributed [3] to the destruction of the phase coherence by quantum phase slips [4, 5].

To understand the superconducting properties of thin wires one has to keep in mind that in an infinite onedimensional conductor all the electronic states are localized. The localization length $\lambda$ in a wire of cross-section $A$ can be estimated as $\lambda \sim k_{F}^{2} A l$, where $k_{F}$ is the Fermi wavevector and $l$ is the mean free path. If the length $L$ of the wire is shorter than $\lambda$, it can be viewed as a metal grain which becomes a good superconductor if the gap $\Delta$ exceeds the quantum level spacing $E_{F} / k_{F}^{3} A L$. On the other hand, a wire of length $L \gg \lambda$ cannot be viewed as a good conductor, and its superconducting properties may be affected by localization.

If the attractive interactions between the electrons are weak, so that the bulk superconducting gap $\Delta$ is small compared to the level spacing $\delta_{\lambda} \sim E_{F} / k_{F}^{3} A \lambda$ of a piece of the wire of length $\lambda$, the superconductivity is suppressed on a microscopic scale. In the more interesting regime of $\Delta \gg \delta_{\lambda}$, the superconducting gap is not affected by the localization 11. The important question in this regime is that of phase coherence between the different parts of the wire. Experimentally this issue can be studied by measuring the persistent current in a nanowire ring as a function of the magnetic flux piercing it. The magnitude of the persistent current oscillations as a function of the flux is a direct measure of the superconducting phase coherence throughout the wire.

In this paper we study the dependence of the persistent current in a superconducting nanoring as a function of the flux and the size of the ring. If the wire is relatively thick, the electrons move freely between different parts of the wire, and therefore the superconducting phase $\phi$ is a well defined classical variable. At low temperatures $T \ll \Delta$ there are no quasiparticle excitations in the nanoring. The allowed states of the system differ by the phase change $\varphi=\phi(L)-\phi(0)$ accumulated over the circumference of the ring. At a given value $\Phi$ of the magnetic flux through the ring $\varphi$ can assume the values $\varphi_{m}=2 \pi \Phi / \Phi_{0}+2 \pi m$, where $m$ is an arbitrary integer, and $\Phi_{0}=\pi \hbar c / e$ is the superconducting flux quantum. The energies of these states are given by

$$
E_{m}=\frac{2 \pi^{2} \hbar^{2} n_{s} A}{m^{*} L}\left(\frac{\Phi}{\Phi_{0}}+m\right)^{2} .
$$

Here $n_{s}$ is the density of superconducting electrons, $m^{*}$ is the electron mass. The dependences of the energy levels (11) on the flux are shown by solid line in Fig. 11(a). The persistent current can be found as a derivative of the ground state energy $I=c d E / d \Phi$ and shows the characteristic sawtooth behavior, Fig. 11(b).

The above picture fails in thin wires, where the fluctuations of the superconducting order parameter cannot be neglected. The most important type of the fluctuations is the quantum phase slip [1, 5] that changes the phase $\phi$ at a point $x$ by $\pm 2 \pi$. We will show that the effect of the rare phase slips on the system reduces to quantum transitions between the levels (11). Such transitions are most important near the degeneracy points at half-integer values of $\Phi / \Phi_{0}$ and result in the small level splitting shown by dashed line in Fig. 11(a). We will also show that the multiple quantum phase slips in longer and thiner wires eventually lead to a crossover from sawtooth to sinusoidal behavior of the persistent current, see dotted line in Fig. 1(b).

The theory of quantum phase slips in nanowires [4, 河] is rather complex. In particular, the most important quantitative parameter describing the phase slip - its action- 

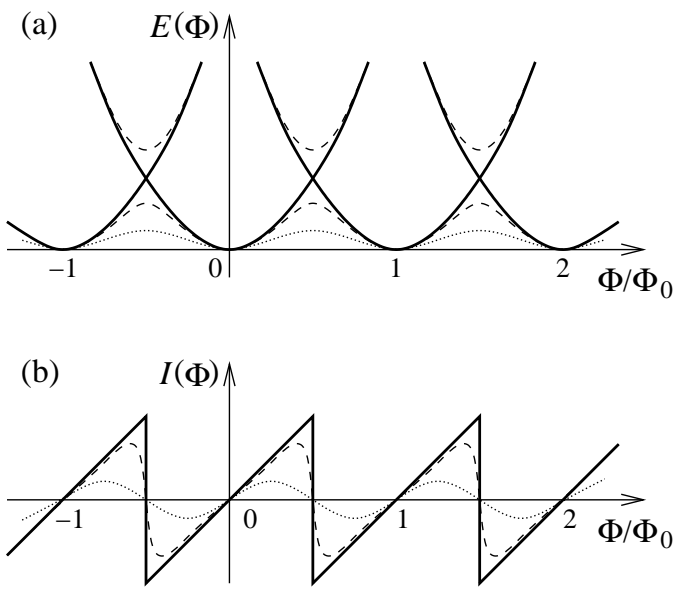

FIG. 1: (a) The energy of a nanoring as a function of the flux $\Phi$ through it. In the limit of relatively large cross-section $A$ the energy shows the classical behavior (11) shown by solid line. Quantum phase slips result in level splitting shown in dashed line, and then lead to sinusoidal dependence of the ground state energy on the flux (dotted line). (b) The persistent current in the nanoring, $I(\Phi)=c d E / d \Phi$. In the classical limit the current shows sawtooth behavior shown by solid line. As the wire becomes thiner, the sawtooth is rounded and eventually transforms to a sinusoidal oscillation.

can be found analytically only up to an unknown numerical coefficient. On the other hand, the effect of the phase slips on the ground state of the nanoring can be studied using a much simpler model of a chain of coupled superconducting grains considered below. Similarly to the nanowire, the chain of grains will have quantum phase slips that affect the persistent current in the same way. We will discuss the relation between the two models in more detail below.

We consider a model of the chain of superconducting grains defined by the following imaginary-time action:

$$
S=\int_{0}^{\beta} d t \sum_{n=1}^{N}\left\{\frac{\dot{\theta}_{n}^{2}}{2 E_{C}}+E_{J}\left[1-\cos \theta_{n}(t)\right]\right\} .
$$

The grains are assumed to be connected by tunnel junctions with Josephson coupling energy $E_{J}$; the capacitance of each junction $C$ gives rise to the charging energy $E_{C}=(2 e)^{2} / C$; the variable $\theta_{n}$ is the phase difference across the $n$-th junction; $\beta=1 / T$. To model a closed chain pierced by flux $\Phi=(\varphi / 2 \pi) \Phi_{0}$, one should impose an additional condition

$$
\sum_{n=1}^{N} \theta_{n}(t)=\varphi
$$

on the phases $\theta_{n}$. Note that the action (2) does not include the charging energy terms due to the selfcapacitance of the grains. These terms, characterized by charging energy $\tilde{E}_{C}$ per grain, are known [6] to give rise to a Kosterlitz-Thouless type quantum phase transition in an infinite chain of Josephson junctions at $E_{J} / \tilde{E}_{C} \sim 1$. The use of model (2) is motivated by the fact that the electric field is very well screened in metal wires which corresponds to the condition $\tilde{E}_{C} \gg E_{J}, E_{C}$.

The ground state properties of the chain can be derived from the partition function $Z=\int e^{-S} D \theta_{n}$ in the limit $\beta \rightarrow \infty$. In this paper we consider the case of strong coupling between the grains $E_{J} \gg E_{C}$. At $E_{C}=0$ the phases $\theta_{n}$ become classical variables, and the energy states of the chain can be found by minimizing the sum of the Josephson energy terms in the action (2) with the constraint (3). At large number of contacts $N \gg 1$ one finds

$$
E_{m}=\frac{E_{J}}{2 N}(\varphi+2 \pi m)^{2},
$$

in complete analogy with Eq. (11) for a metal ring.

At finite small $E_{C}$ the fluctuations of $\theta_{n}(t)$ should be taken into consideration. First, one can include the Gaussian fluctuations around the classical solutions $\theta_{n}(t)=$ const. This is accomplished by expanding the cosine in the action (2) up to quadratic terms in $\theta_{n}$. The resulting correction to the ground state energy $\delta E_{m}=$ $(N-1) \sqrt{E_{J} E_{C}}$ accounts for the zero-point oscillations of the particles in the minima of the cosine potential. This constant correction does not affect the shape of the oscillations of the persistent current $I=(2 e / \hbar) d E / d \varphi$. One can also include the anharmonic terms of the expansion of the cosine potential in the action (2). The resulting corrections yield a small in $E_{C} / E_{J}$ distortion of the sawtooth dependence $I(\Phi)$, but do not significantly affect the discontinuities at half-integer $\Phi / \Phi_{0}$.

A more interesting fluctuation of the phases $\theta_{n}(t)$ involves an instanton (quantum phase slip), i.e., a trajectory that begins near one of the minima (4) of the potential energy in action (2) at $t=0$ and ends near another minimum at $t=\beta$. For instance, a trajectory starting at $\theta_{n}(0)=\varphi / N$ and ending at $\theta_{n}(\beta)=(\varphi-2 \pi) / N+2 \pi \delta_{n k}$ for arbitrary $k$ in the interval $1 \leq k \leq N$ connects the minima (4) with $m=0$ and $m=-1$. The shape of the instanton trajectory can be found by minimizing the classical action (2) with the above boundary conditions on $\theta_{n}(t)$. In the case of large number of junctions $N \gg 1$ the dominant contribution is due to the contact $k$ where the phase slip occurs, and the contributions of the other contacts can be neglected. Then one obtains the usual result

$$
\theta_{k}(t)=4 \arctan \exp \left[\sqrt{E_{J} E_{C}}\left(t-t^{\prime}\right)\right],
$$

where $t^{\prime}$ is the arbitrary moment in time where the phase slip is centered; we have assumed the limit of low temperature $T \ll \sqrt{E_{J} E_{C}}$. The action associated with this instanton trajectory is $S_{0}=8 \sqrt{E_{J} / E_{C}}$, 12].

The instantons account for the possibility of the system tunneling between the different minima (4) of the 
potential energy in action (2). The effect of the instantons on the ground state energy can be accounted for by considering a tight-binding Hamiltonian defined as

$$
H \psi_{m}=E_{m} \psi_{m}-N v\left(\psi_{m-1}+\psi_{m+1}\right) .
$$

Here $\psi_{m}$ is the amplitude of the system at the state $m$ with energy $E_{m}$ given by Eq. (4). The hopping matrix element is exponentially small,

$$
v=\frac{4}{\sqrt{\pi}}\left(E_{J}^{3} E_{C}\right)^{1 / 4} \exp \left(-8 \sqrt{\frac{E_{J}}{E_{C}}}\right),
$$

where the exponent coincides with the instanton action $S_{0}$, and the prefactor can be obtained by considering the problem (2) in the case of a single junction (without the constraint (30).

It is important to note that for any $k$ the set of phases $\theta_{n}=(\varphi-2 \pi) / N+2 \pi \delta_{n k}$ describes the same physical state of the chain. Thus the hopping matrix elements due to the instantons (5) in all the $N$ junctions must be summed up, resulting in the additional factor of $N$ in the hopping term in the Hamiltonian (6).

At $N v \ll E_{J} / N$ the hopping term is small compared with the diagonal matrix elements in the Hamiltonian (6). Its effect is most significant when $\Phi / \Phi_{0}$ is half-integer, and the energy levels $E_{m}$ are degenerate, Fig. 1(a). In this regime hopping gives rise to the level repulsion shown by the dashed line in Fig. 1 1(a) and the respective rounding of the sawtooth in current, Fig. 1(b). The shape of the current steps is given by

$$
I=\frac{2 e E_{J}}{\hbar N}\left[\chi-\frac{\pi \chi}{\sqrt{\chi^{2}+\left(v N^{2} / \pi E_{J}\right)^{2}}}\right],
$$

where $\chi=\varphi-\pi=2 \pi\left(\Phi / \Phi_{0}-1 / 2\right) \ll 1$.

In general hopping affects the spectrum of the Hamiltonian (6) dramatically. To find the ground state energy $E(\varphi)$ it is more convenient to apply the Hamiltonian (6) to the wavefunction in "coordinate representation," $\psi(x)=\sum_{m} \psi_{m} e^{i(2 m-\varphi / \pi) x}$. The resulting Schrödinger equation then takes the form of Mathieu equation:

$$
\psi^{\prime \prime}(x)+(a-2 q \cos 2 x) \psi(x)=0,
$$

where parameter $a=2 N E / \pi^{2} E_{J}$ is proportional to the energy $E$, and $q=N^{2} v / \pi^{2} E_{J}$. The phase $\varphi$ enters the problem via a twisted boundary condition $\psi(x+\pi)=$ $e^{-i \varphi} \psi(x)$. The regime of strong hopping in the Hamiltonian (6) corresponds to $q \gg 1$. In this case the dependence of the eigenvalue $a$ on the phase $\varphi$ is exponentially small, $a=-16 \sqrt{2 / \pi} q^{3 / 4} e^{-4 \sqrt{q}} \cos \varphi$, Ref. 7 . For the persistent current $I=(2 e / \hbar) d E / d \varphi$ we then find

$$
I=16 \sqrt{2 N} \frac{e}{\hbar}\left(E_{J} v^{3}\right)^{1 / 4} \exp \left(-\frac{4}{\pi} N \sqrt{\frac{v}{E_{J}}}\right) \sin \varphi .
$$

It is important to note that the Schrödinger equation for the Hamiltonian (6) coincides with Eq. (9) at arbitrary hopping strength $q$. Thus the problem of the flux dependence of the persistent current reduces to the solving the well known Mathieu equation (9) with the appropriate twisted boundary conditions. This conclusion is our main result. The shape of the current oscillations is controlled by a single parameter $q$. As $q$ changes from 0 to $\infty$, the shape crosses over from sawtooth to the sinusoidal behavior.

The Hamiltonian (6) has been rigorously derived for the model (2) of a chain of coupled superconducting grains. Although nanorings are not described by this model, the essential low-energy physics of a superconducting state being destroyed by quantum phase slips remains the same, and the Hamiltonian (6) and the respective Schrödinger equation (9) are still valid.

To apply the Hamiltonian (6) to superconducting nanorings, one needs to relate the matrix elements $E_{m}$ and $N v$ to the parameters describing the nanowires, such as their length $L$, coherence and localization lengths $\xi$ and $\lambda$, etc. The diagonal matrix elements $E_{m}$ are given by their classical values (11). The important difference between nanowires and chains of superconducting grains appears in evaluating the hopping matrix element $N v$. In the absence of tunneling barriers the quantum phase slips must involve suppression of the superconducting gap $\Delta$ in the wire. The typical volume of space where the gap is suppressed is $V \sim A \xi$. Given the condensation energy per unit volume $\varepsilon \sim m^{*} k_{F} \Delta^{2}$ and the typical correlation time $\tau \sim 1 / \Delta$, one can estimate the phase slip action in the wire $S_{0} \sim \varepsilon V \tau \sim \sqrt{\Delta / \delta_{\lambda}} \sim \lambda / \xi$. A more rigorous treatment $[4,5]$ of the quantum phase-slips gives rise to the same result, but unfortunately the numerical coefficient cannot be obtained analytically. Taking into account the obvious analogy between the number of Josephson contacts $N$ and the length $L$ of the wire, we conclude $N v \propto L e^{-S_{0}}$, with $S_{0} \sim \lambda / \xi$.

Another feature of realistic nanowires not included in our model of a chain of Josephson junctions is the mesoscopic fluctuations introduced by the disorder. For instance, since the instanton action depends on the mean free path, the fluctuations of the random potential can lead to the fluctuations of $S_{0}$. This effect should lead to mesoscopic fluctuations of the persistent current; the magnitude of the fluctuations is expected to be small in relatively thick wires.

A more subtle effect of the random potential affects the relative phases of instantons in different parts of the wire. It can be explored within our model by adding gates to the grains and applying random gate voltages. It has been shown recently [8, 9] that the gate voltages give rise to Aharonov-Casher interference effects for the instantons in different junctions, and under certain conditions can suppress the effects of the phase slips.

To account for the effect of the random gate potential 
on the persistent current, we present the electrostatic charging energy of the chain as

$$
H_{C}=\sum_{n l} \frac{1}{2}\left[C^{-1}\right]_{n l}\left(Q_{n}-q_{n}\right)\left(Q_{l}-q_{l}\right),
$$

where $\left[C^{-1}\right]_{n l}$ is the matrix of inverse capacitances of the system, $Q_{n}$ is the charge at the $n$-th grain. Parameters $q_{n}$ are determined by the gate voltages and have the meaning of the values of the charges $Q_{n}$ at which the electrostatic energy is minimized.

Using the fact that the grain charge $Q_{n}$ and phase $\phi_{n}$ are conjugate variables and introducing $\theta_{n}=\phi_{n}-\phi_{n+1}+$ $\varphi / N$, one rederives the imaginary-time action (2) with an additional term

$$
\delta S=-i \sum_{n} p_{n} \dot{\theta}_{n}, \quad p_{n}=q_{1}+q_{2}+\ldots+q_{n},
$$

accounting for the gate voltages. Since the instanton (5) corresponds to a trajectory where the phase in contact $k$ changes by $2 \pi$, the respective contribution to the hopping matrix element in the Hamiltonian (6) has an additional phase $e^{i 2 \pi p_{n}}$. Therefore for the total hopping matrix element we obtain $v \sum_{n} e^{i 2 \pi p_{n}}$ instead of $N v$, in agreement with Ref. 8. If the random gate voltages are sufficiently strong, so that $q_{n} \sim 1$, the average of the hopping matrix element in the Hamiltonian (6) vanishes: $\left\langle v \sum_{n} e^{i 2 \pi p_{n}}\right\rangle=0$. Its typical values are of the order of the mean square fluctuation, thus decreasing from $N v$ in the regular case to $\sim \sqrt{N} v$ in the case of random charges. In the limit of large $N$, the same square-root dependence on the length of the chain will result even from weak disorder. This suppression of the effect of phase slips results in a higher value of the persistent current, which can be estimated by replacing $v \rightarrow v / \sqrt{N}$ in Eqs. (8) and (10). The most significant change in the properties of the persistent current caused by the random gate potential is that instead of $\ln I \propto-N$ we find a slower dependence $\ln I \propto-N^{3 / 4}$ on the length of the chain at large $N$.

It is worth noting that the above result cannot be obtained from the action averaged over the random gate potentials $q_{n}$. Indeed, one can easily see that $\langle\delta S\rangle=0$, thereby restoring our previous results $(8)$ and $(10)$. A term similar to (12) also appears in the theory of the persistent current in disordered wires. This term breaks the translational invariance and destroys the zero mode of the instanton action. Thus the conventional argument that the zero mode gives rise to the prefactor of the instanton contributions to the matrix elements proportional to the length of the system does not apply. Consequently we expect the total matrix element in the Hamiltonian (6) to behave as $\sqrt{L} e^{-S_{0}}$, leading to the persistent current $\ln I \propto-L^{3 / 4} e^{-S_{0} / 2}$. Averaging of the action over the disorder would restore the zero mode and lead to the incorrect result $\ln I \propto-L e^{-S_{0} / 2}$.

Our theoretical predictions can be tested in devices similar to those of Refs. 2, 3. Although we discussed the geometry of a nanoring of uniform thickness, all the conclusions are applicable in the case of a straight nanowire with ends shorted by a bulk superconductor. In this geometry only the quantum phase slips in the nanowire part should be considered, and thus the length of the system $L$ is that of the nanowire only. The most interesting theoretical prediction to be tested in such devices is the dependence of the persistent current on the magnetic flux $\Phi$. The dependence $I(\Phi)$ evolves from sawtooth to the sinusoidal one, Fig. 1(b), as the nanowire becomes longer and/or thiner. We expect that the experimentally measured shape of the oscillations of $I(\Phi)$ can be fitted to the function $d a(\varphi) / d \varphi$, determined from the Mathieu equation (9). The only free parameter in such a fit is the effective phase slip strength $q$.

Another type of systems to which our predictions should apply is the one-dimensional arrays of Josephson junctions. Recently it became possible to tune the Josephson coupling energy in such devices by external magnetic field [10]. As a result, one should be able to study the whole crossover from sawtooth to sinusoidal dependence of $I(\Phi)$ in a single sample.

In conclusion, we have studied the persistent current in nanoscale superconducting rings. As the wire becomes thiner, the quantum phase slips begin to suppress the current and change its flux dependence $I(\Phi)$. The latter can be found by solving Mathieu equation (9) with the appropriate boundary condition. The Aharonov-Casher interference suppresses the effects of the phase slips and gives rise to the unusual length dependence $\ln I \propto-L^{3 / 4}$.

KAM acknowledges the support of the Sloan Foundation and NSF Grant DMR-9974435. The work at the University of Minnesota is supported by NSF Grants DMR-9731756 and DMR-0120702. The authors acknowledge the hospitality of Aspen Center for Physics and ITP at UC-Santa Barbara, where part of the work was performed.

[1] See, e.g., the review by J. von Delft and D. C. Ralph, Phys. Rep. 345, 61 (2001).

[2] A. Bezryadin, C. N. Lau and M. Tinkham, Nature 404, 971 (2000).

[3] C. N. Lau, N. Markovic, M. Bockrath, A. Bezryadin, and M. Tinkham, Phys. Rev. Lett. 87, 217003 (2001).

[4] A. D. Zaikin, D. S. Golubev, A. van Otterlo, and G. T. Zimanyi, Phys. Rev. Lett. 78, 1552 (1997).

[5] D. S. Golubev and A. D. Zaikin, Phys. Rev. B 64, 014504 (2001).

[6] R. M. Bradley and S. Doniach, Phys. Rev. B 30, 1138 (1984).

[7] M. Abramowitz and I. A. Stegun, Handbook of Mathematical Functions, (Dover, New York, 1974).

[8] D. A. Ivanov, L. B. Ioffe, V. B. Geshkenbein, and G. Blatter, Phys. Rev. B 65, 024509 (2002).

[9] J. R. Friedman and D. V. Averin, cond-mat/0109544. 
[10] E. Chow, P. Delsing, and D. B. Haviland, Phys. Rev. Lett. 81, 204 (1998).

[11] The ratio $\delta_{\lambda} / \Delta$ coincides with the Ginzburg parameter Gi. In this paper we concentrate on the regime of $\mathrm{Gi} \ll 1$.

[12] At non-zero self-capacitance of the grains one finds a correction $\tilde{S} \sim\left(E_{J} / \tilde{E}_{C}\right)^{1 / 2} \ln N$. In the limit $N \rightarrow \infty$ it leads to the superconductor-insulator transition [6], but in finite systems our results for $I(\Phi)$ remain valid. Furthermore, at $\tilde{E}_{C} \gg E_{C}$ the correction $\delta S$ is negligible unless $N$ is exponentially large. 